\title{
Chirurgin, Unternehmerin, Pionierin der Krankenpflege
}

\section{Verena E. Müller}

lic. phil., Historikerin, Zürich

Am ersten Schweizerischen Frauenkongress in Genf 1896 forderte die 33-jährige Ärztin Anna Heer (1863-1918) eine Reform der Pflegeausbildung. Dazu war ein eigenes Krankenhaus mit angeschlossener Unterrichtsstätte nötig. Nur fünf Jahre später öffnete in Zürich die «Schweizerische Pflegerinnenschule mit Spital» ihre Tore. In jener Zeit waren Schweizerinnen ohne politische Rechte, Ehefrauen finanziell unmündig. Vor diesem Hintergrund war das Spital «von Frauen für Frauen» eine Sensation.

«Mein Vater hatte eine kleine Fabrik und eine grosse Familie.» So deutete Anna Heer 1900 komplexe Familienverhältnisse an. Geboren wurde sie 1863 in Olten. Ihr Vater, der Thurgauer Isaak Heer, war Schuhfabrikant. 1877 zog die Familie nach Suhr AG. Nun produzierte

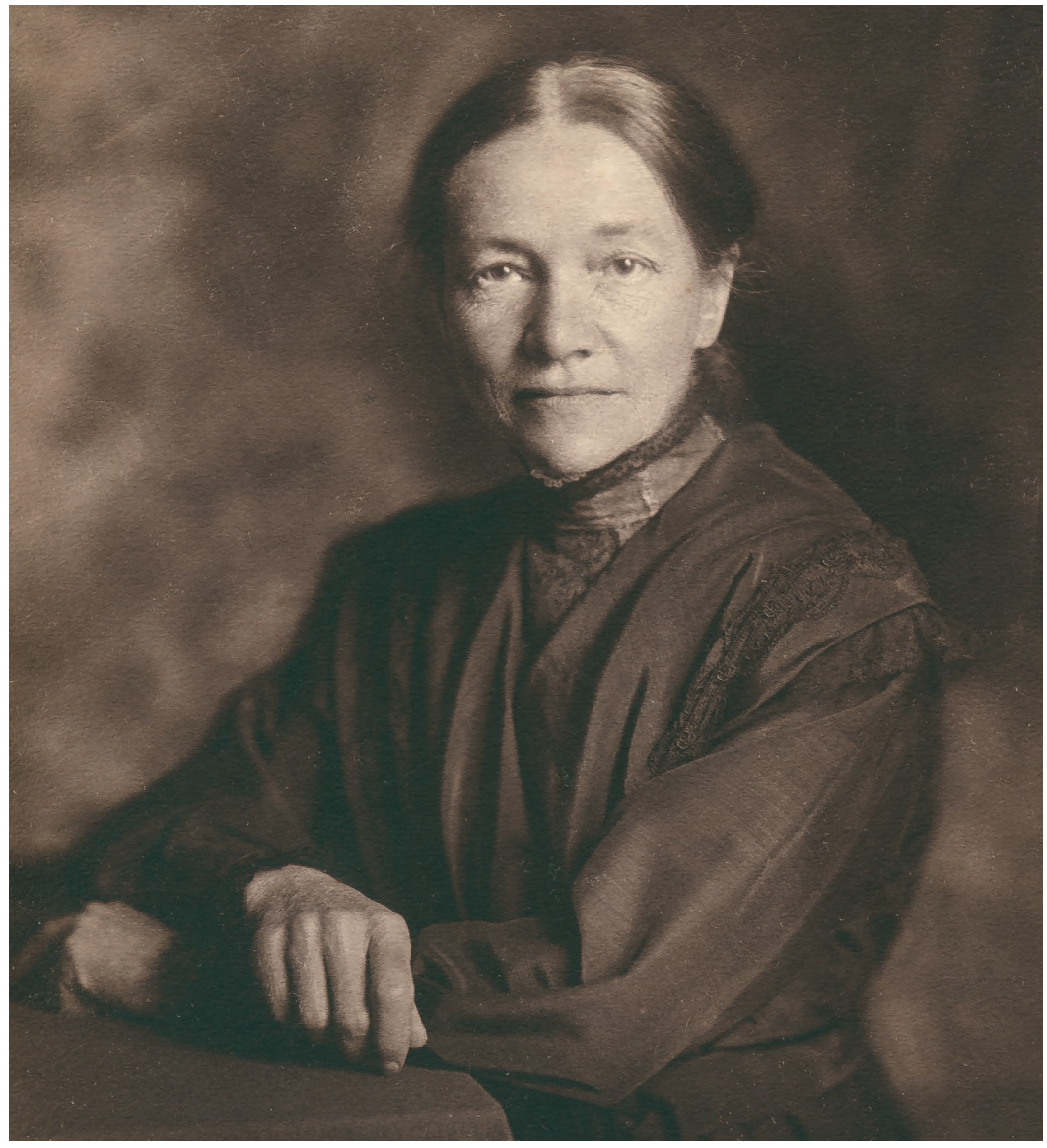

Abbildung 1: Anna Heer.
Heer elastische Gewebe, doch auch damit hatte er kaum Erfolg. Von Annas Mutter, der Oltner Bürgerin Josephine Heer-Klein, ist kaum etwas überliefert. Sie gebar elf Kinder, sieben erreichten das Erwachsenenalter.

\section{Kunstgewerbe- und Höhere Töchter- schule}

Wie Aargauer Prozessprotokolle zeigen, war Isaak Heer eine problematische Persönlichkeit, aber die drei älteren Töchter erhielten eine solide Allgemeinbildung. Nach Abschluss der Mädchenbezirksschule schrieb sich Anna Heer 1880 an der eben gegründeten Zürcher Kunstgewerbeschule ein. Eine Aarauer Freundin vermittelte dem Mädchen Kost und Logis beim kinderlosen Ehepaar Kaspar und Bertha Grob, die allmählich zu ihren Pflegeeltern wurden. Bei Annas Einzug war Sekundarlehrer Grob bereits Erziehungssekretär des Kantons Zürich. Er ermunterte sie, von der Kunstgewerbeschule an die Höhere Töchterschule zu wechseln. Ohne die Begegnung mit den Grobs ist Anna Heers Karriere undenkbar. Kaspar Grob war nicht nur ihr Mentor. Dank seines engmaschigen Beziehungsnetzes konnte er Annas Pläne regelmässig direkt und indirekt fördern. 1901 vererbte er ihr die Liegenschaft am Rande der Zürcher Altstadt. Hier, an den Unteren Zäunen 17, hatte sie ihre private Praxis, da wohnte sie bis 1918.

\section{Studium in Zürich, Studienaufenthalt in London}

Vom Sommersemester 1883 bis 1888 studierte Anna Heer an der Universität Zürich Medizin. Nur fünf der 


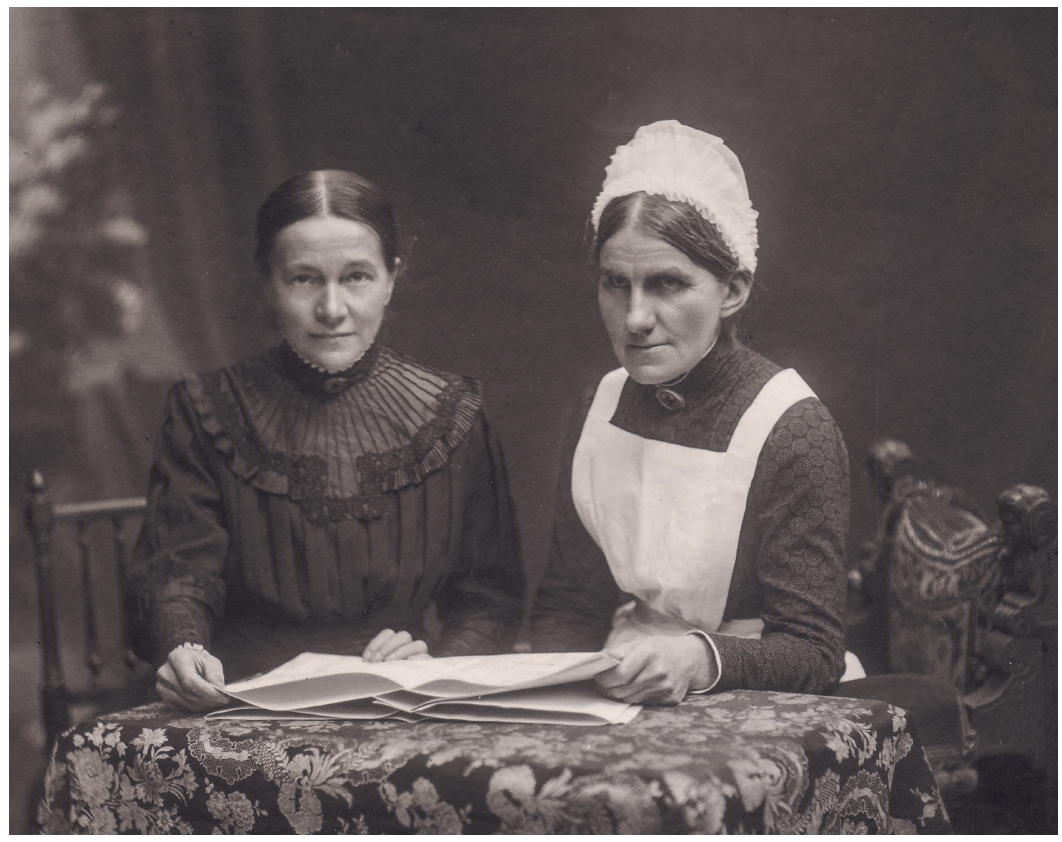

Abbildung 2: Anna Heer und Ida Schneider.

33 Medizinstudentinnen kamen aus der Schweiz. Ihr Tag begann morgens um halb fünf. Auf das «Examensgespenst» des Propädeutikums folgten die klinischen Semester, anschliessend die Schlussprüfung. Dann verbrachte sie einige Monate in London, damals die Metropole eines Weltreichs. Spätestens da hörte sie von der Reform der Krankenpflege, die Florence Nightingale und die British Association of Nurses propagierten.

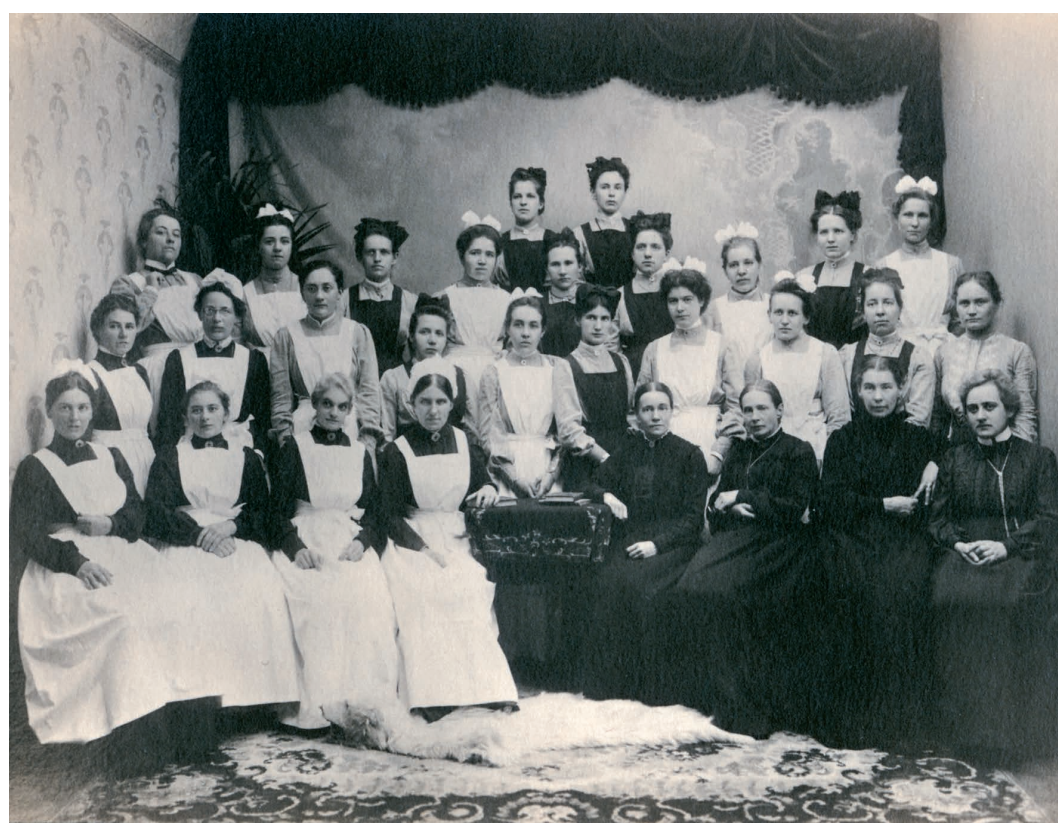

Abbildung 3: Links vom Tisch: Oberin Schneider. Rechts: Anna Heer, Marie HeimVögtlin, Jenny Thomann-Koller.

\section{Praxis und Familiäres}

Zurück in Zürich, vertrat Anna Heer ab Februar 1889 die erste Schweizer Ärztin Marie Heim-Vögtlin während ihres Mutterschaftsurlaubs. Anders als andere frühe Medizinerinnen interessierte sich Anna Heer weniger für Innere Medizin, Frauen- und Kinderheilkunde. «Wenn sich meine Hand geschickt genug erweisen wird, soll die Chirurgie eine Hauptrolle bei mir spielen", schrieb die Studentin. Ihre Dissertation «Über Schädelbasisbrüche» reichte sie 1892 bei Ulrich Krönlein ein. 1889 verlegte Familie Heer ihren Wohnsitz von Suhr nach Zürich. Ob sie beabsichtigte, künftig auf Kosten der Tochter zu leben, ist unklar. Anna und ihre Schwester Maria, später Romanistin, blieben jedenfalls beim Ehepaar Grob. Isaak und Josephine Heer wohnten ab ca. 1895 an unterschiedlichen Adressen, damals ein ungewöhnlicher Schritt. Drei Geschwister blieben bei der Mutter, Anna fiel immer mehr die Rolle des Familienvorstandes zu.

\section{Freundschaft mit Ida Schneider}

In einer Privatklinik, dem «Schwesternhaus vom Roten Kreuz», betreute Anna Heer einige Patientinnen. Hier begegnete sie 1892 der sechs Jahre jüngeren Krankenschwester Ida Schneider. Als Tochter des Professors für Römisches Recht lebte sie in einem kultivierten, mittelständischen Milieu. Die beiden Frauen wurden Freundinnen und zum verschworenen Arbeitsteam. Bis zu Anna Heers Tod blieb Ida Schneider ihre engste Mitarbeiterin und Vertraute.

\section{Anna Heer und der Schweizerische Gemeinnützige Frauenverein SGF}

Zwei Jahre vor dem Genfer Kongress hatte Anna Heer an der Jahresversammlung des Schweizerischen Gemeinnützigen Frauenvereins 1894 ihre Vision vorgestellt. Noch erhielten einzig Nonnen oder Diakonissen eine fundierte Ausbildung in Pflege. Nach englischem Vorbild wollte Anna Heer bürgerlichen Töchtern den Zugang zum Beruf ohne religiöse Verpflichtung ebnen. Ihr zweites Kernanliegen war die wirtschaftliche Besserstellung der Pflegefachleute. Dies wollte sie mit der Gründung eines Berufsverbandes erreichen. - Gelegentlich trübten Missverständnisse Anna Heers Verhältnis zum SGF, zu gewagt erschien einzelnen Mitgliedern das Projekt. Dennoch waren aus diesem Kreis Hunderte bereit, beträchtliche Summen zu sammeln oder gar selber zu spenden. Ohne die Unterstützung durch den SGF wären ihre Pläne ein Traum geblieben. 


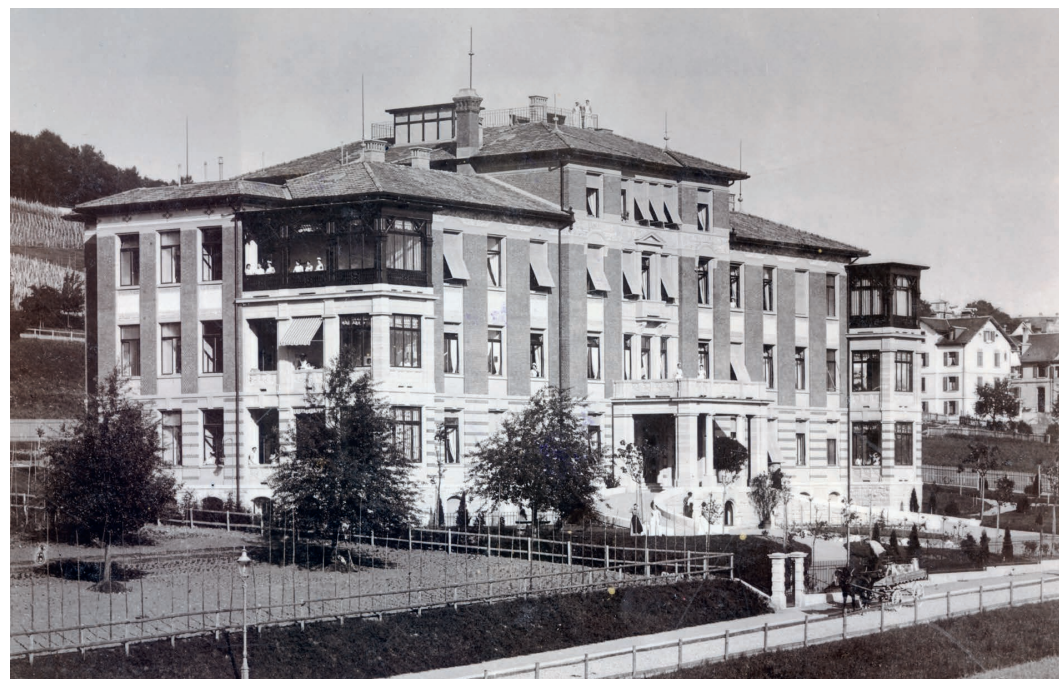

Abbildung 4: Pflegerinnenschule, ca. 1901

\section{Unterwegs zum eigenen Spital}

Während Isaak Heer ein eher glückloser Einzelkämpfer war, verstand es die Tochter, viele - auch unbekannte Frauen und einige einflussreiche Männer für ihre Ideen zu gewinnen. Einzig in die Baukommission berief sie Männer. Neben Anna Heer und Ida Schneider sassen in diesem Gremium Pflegevater Grob, inzwischen Stadtrat geworden, die Professoren Schneider und Heim, Seidenkaufmann Johann Spörri sowie Kantonsbaumeister Hermann Fietz, der gleichzeitig den Bau der neuen aseptischen Operationsanlage des Kantonsspitals führte. Entscheidend war ferner die Mitarbeit Marie HeimVögtlins. Ihr Prestige veranlasste manche Mäzene zu Grosszügigkeit. Bis kurz vor ihrem Tod war sie Mitglied des Ausschusses, nach der Eröffnung des Spitals betreute sie die «Kinderstube». Zu ihren Ehren fand die Grundsteinlegung 1899 an ihrem 25. Doktorjubiläum statt. Eröffnet wurde die "Pflegerinnenschule» am 30. März 1901, erste Schülerinnen traten ein, Ida Schneider wurde Oberin, Anna Heer Chefärztin. Beide arbeiteten für die «Pflegi» stets ohne Gehalt, weshalb für Anna Heer die Weiterführung ihrer Privatpraxis von existenzieller Bedeutung war. - Als Vorstufe zur geplanten Organisation des Pflegepersonals eröffneten Anna Heer und Ida Schneider 1899 eine Arbeitsvermittlungsstelle für Krankenwärterinnen und -wärter. Ab 1901 war diese der Pflegerinnenschule angegliedert und wurde von den Zürcher Behörden offiziell gefördert.

Korrespondenz: lic. phil. Verena E. Mülle Culmannstrasse 19 CH-8006 Zürich vemueller[at]gmail.com

\section{Anna Heer, Walter Sahli und der Schweizerische Krankenpflegebund}

Die Frage der Ausbildungsreform beschäftigte neben Anna Heer den Berner Arzt Walter Sahli. Ihm gelang es, seine Pläne schneller umzusetzen. 1899 gründete er die Rotkreuzschule für Krankenpflege am Berner Lindenhofspital, 1903 übertrug die Eidgenossenschaft dem Schweizerischen Roten Kreuz die Zuständigkeit für die Ausbildung des Pflegepersonals, ab 1906 führte Sahli das Generalsekretariat des SRK. Trotz einer gewissen Rivalität verfolgten Heer und Sahli gemeinsame Ziele und gründeten zusammen 1910 den Schweizerischen Krankenpflegebund, den Anna Heer bis 1916 präsidierte.

\section{Anna Heers Kollegen}

Berufskollegen begegneten Anna Heer skeptisch; dennoch kam es nicht nur mit Walter Sahli in einzelnen Fällen zu fruchtbarer Zusammenarbeit. Theodor Wyder, Professor für Gynäkologie und Chef der Universitätsklinik, war kein Freund des Frauenstudiums. Er befürchtete zudem, das künftige Spital würde Patientinnen von seiner Frauenklinik abwerben. Trotz dieser Vorbehalte liess er sich davon überzeugen, dass sich die sorgfältige Ausbildung insbesondere der «Vorgängerinnen» (Wochenbettpflege) aufdrängte. Schliesslich durfte sich Anna Heer bei ihm zwei Monate lang als «Volontärarzt» in medizinischen Belangen und Spitalführung weiterbilden. Bestens verliefen die Kontakte zum Zürcher Stadtarzt Krucker. Carl Schuler, Chirurg an der katholischen Privatklinik Theodosianum, war in den ersten Jahren regelmässig bei schwierigen Operationen in der «Pflegi» im Einsatz.

Die zunächst erfolgreiche «Pflegerinnenschule» stand während des Ersten Weltkriegs vor grossen, auch finanziellen Herausforderungen. Als sich Anna Heer während einer Operation im November 1918 verletzte und einen Monat später an einer Sepsis starb, hinterliess sie ein belastetes Erbe. Nach einigen Turbulenzen erholte sich die Institution jedoch wieder und existierte bis zum Ende des 20. Jahrhunderts.

\section{Bildnachweis}

Gosteli-Archiv, Worblaufen

\section{Literatur}

1 Baumann Kurer S. Die Gründung der Schweizerische Pflegerinnenschule mit Frauenspital in Zürich 1902 und ihre Chefärztin Anna Heer (1863-1918). Zürich 1991.

2 Benz E. Anna Heer. In: Schweizerische Lehrerinnen-Zeitung. Band 23 (1918-1919), Heft 6, S. 118-24.

3 Braunschweig S, Francillon D. Professionelle Werte pflegen. 1910-2010. 100 Jahre SBK. Schweizer Berufsverband der Pflegefachfrauen und Pflegefachmänner (SBK). Zürich 2010.

4 von Segesser A. Dr. med. Anna Heer (1863-1918). 1948.

5 Studer-von Goumoëns E. 25 Jahre Schweizerische Pflegerinnenschule mit Frauenspital in Zürich 1901-1926. Zürich 1926.

6 Im März 2019 erscheint im eFeF-Verlag eine neue Biographie Anna Heers von Verena E. Müller. 